\title{
Promoting CPAP adherence in obstructive sleep apnea
}

\author{
Moussa Riachy* \\ Pulmonary and Critical Care Department, Hotel Dieu de France Hospital, Beirut, Lebanon
}

Continuous positive airway pressure (CPAP) remains the gold standard therapeutic intervention in obstructive sleep apnea (OSA) [1]. Significant adherence issues with CPAP (defined as device use for $<4$ hours/night) ', reported to range from $29-83 \%$ in OSA patients', decrease its effectiveness in long-term therapy [2].

In a cohort study conducted at the Sleep Center of Hôtel-Dieu de France Hospital in Lebanon over 8 years, the CPAP adherence was studied. Median adherence time was 957 days. It also highlighted an adherence of $70.3 \%$ at 6 months and $49.3 \%$ at 8 years [3].

Among the factors that were found to affect adherence to CPAP, Riachy et al. [3] found a faster short term (first 3 months) followed by a slower long term dropped out. The identification of true predictors of adherence to CPAP, independent of the best provider care, is of such great importance since it helps optimize the care of OSA patients with a more customized approach [1]. This could help in developing individualized plans for different patients' profiles in order to optimize CPAP adherence at each moment of the follow-up of his treatment.

In the beginning of the CPAP treatment, the oxygen desaturation index (ODI) increase of 10 per hour at baseline was associated with a $20 \%$ decrease in the risk of short-term CPAP non-adherence $(\mathrm{HR}=$ 0.98). This decrease is consistent with the results of Kohler et al. [4]. In contrast to our results, McArdle et al. [5] showed that it was rather the daytime sleepiness (ESS) at diagnosis that affected short-term adherence.

The use of a nasal mask reduced the risk of non-adherence by $50 \%$ with an HR of 0.48 . Therefore, suggesting a nasal mask preferentially in patients starting CPAP appears warranted and justified $[6,7]$.

On the long term, buying the CPAP device in a health system that emphasizes the patient participation seems to play an important role in adherence to treatment. The risk of non-adherence is reduced by 5.5 times compared to renting it. There are several postulates for this influence including the patient's conviction of long-term need for the device in order to maximize benefits both symptomatically and physiologically [3]. In addition, the number of follow-up contacts by the provider, and self-reported improvement on treatment were positive predictors of long-term adherence. Each contact with the service provider reduced the risk of non-adherence to CPAP by $18 \%$ $(\mathrm{HR}=0.847)$, marking the importance of a patient support program that could promote education on the optimal use of the CPAP. Spouse encouragement plays also an important role [3]. This was highlighted in a meta-analysis by Wozniak et al. [8] that showed that support interventions were linked to slightly better adherence.

Long-term adherence to CPAP is related to the patient's weight change on diet. Our study showed that patients on a diet plan were 4.8 times more likely to stop using the CPAP. This could be interpreted by an improvement of the OSA symptoms after weight loss even without a complete recovery from the underlying condition.

\section{References}

1. Sullivan CE, Issa FG, Berthon-Jones M, Eves L (1981) Reversal of obstructive sleep apnoea by continuous positive airway pressure applied through the nares. Lancet: 862865. [Crossref]

2. Weaver TE, Grunstein RR (2008) Grunstein, Adherence to continuous positive airway pressure therapy: the challenge to effective treatment. Proc Am Thorac Soc 5: 173-178. [Crossref]

3. Riachy M, Najem S, Iskandar M, Choucair J, Ibrahim I, et al. (2017) Factors predicting CPAP adherence in obstructive sleep apnea syndrome. Sleep Breath 21: 295-302. [Crossref]

4. Kohler M, Smith D, Tippett V, Stradling JR (2010) Predictors of long-term compliance with continuous positive airway pressure. Thorax 65: 829-832. [Crossref]

5. McArdle N, Devereux G, Heidarnejad H, Engleman HM, Mackay TW, et al. (1999) Long-term use of CPAP therapy for sleep apnea/hypopnea syndrome. Am J Respir Crit Care Med 159: 1108-1114. [Crossref]

6. Mortimore IL, Marshall I, Wraith PK, Sellar RJ, Douglas NJ (1998) Neck and tota body fat deposition in nonobese and obese patients with sleep apnea compared with that in control subjects. Am J Respir Crit Care Med 157: 280-283. [Crossref]

7. Borel JC, Tamisier R, Dias-Domingos S, Sapene M, Martin F, et al. (2013) Type of mask may impact on continuous positive airway pressure adherence in apneic patients. PLoS One 8: e64382. [Crossref]

8. Lichuan Ye, Atul Malhotra, Karen Kayser, Danny G. Willis, June Horowitz, et al (2015) Spousal involvement and CPAP adherence: a dyadic perspective. Sleep Med Rev 19: 67-74. [Crossref]
Copyright: C2017 Riachy M. This is an open-access article distributed under the terms of the Creative Commons Attribution License, which permits unrestricted use, distribution, and reproduction in any medium, provided the original author and source are credited.
Correspondence to: Moussa Riachy, Pulmonary and Critical Care Department, Hotel Dieu de France Hospital, Beirut, Lebanon, E-mail: riachy@dm.net.lb

Received: April 03, 2017; Accepted: April 24, 2017; Published: April 26, 2017 